\title{
Bacterial chemotaxis
}

\section{Recommended papers:} A modular gradient-sensing network for chemotaxis in Escherichia coli
revealed by responses to time-varying stimuli. T.S. Shimizu, Y. Tu, H.C. Berg. Molecular Systems Biology 6, 382, 2010.

Thermal Robustness of Signaling in Bacterial Chemotaxis. O. Oleksiuk, V. Jakovlievic, N. Vladimirov, R. Carvalho, E. Paster, W.S. Ryu, Y. Meir, N.S. Wingreen, M. Kollmann, V. Sourjik. Cell 145, 312-321, April 2011.

Recommended with a commentary by M. Vergassola, Inst. Pasteur \& CNRS, Paris.

The motion of bacteria is driven by a variety of environmental stimuli, such as light (phototaxis), electric and magnetic fields (galvano- and magneto-taxis), temperature (thermotaxis) and concentration of chemicals (chemotaxis). The latter is perhaps the most studied case and constitutes a paradigmatic example of signal transduction pathway, coupling the interior of the cell with the external environment. There is a long-standing tradition of physicists contributing to the field, as evidenced by its classical references [1,2]. Past years have brought major progress in our understanding of the chemotaxis molecular pathway, both at the experimental and at the modeling level, as exemplified by the recent papers recommended here.

The bacterium most considered for chemotaxis studies is Escherichia coli, which is covered by multiple flagella, about $20 \mu \mathrm{m}$ long and $20 \mathrm{~nm}$ thick. Flagella are attached to the body of the bacterium via flagellar motors, which are driven by the transmembrane ion gradient, reaching rotation speeds on the order of $100 \mathrm{~Hz}$. Rotation of the flagella is converted into propulsion of the bacterium due to the cylinder-like shape of the flagella. Low Reynolds number fluid dynamics [3] demonstrates indeed that the difference in drag between parallel and perpendicular motions of a cylinder generates a propulsive force. Flagella are chiral, so that they can assemble into a bundle when rotating in a certain direction (counterclockwise for E. coli) but they cannot when one or several of them rotate clockwise [4]. Rotations counter-clockwise/clockwise of the flagella correspond to the phases of run and tumble observed at the microscope when looking at $E$. coli swimming. Runs last for periods of about $1-2 \mathrm{~s}$ and the bacterium swims at about $20 \mu \mathrm{m} / \mathrm{s}$ in standard conditions. Tumbles are much shorter, lasting only fractions of a second; they are similar to a scattering event, with the incoming and the outgoing swimming directions almost ${ }^{1}$ uncorrelated. The motion of the bacterium on time scales longer than a few seconds (the microscopic de-correlation time) is thus analogous to a random walk.

In the presence of chemoattractants, $E$. coli is able to orient and climb gradients via the chemotaxis pathway. The basic mechanism of orientation is based on timecomparisons: the local concentration is compared to that in the past (few seconds

\footnotetext{
1 The two directions are in fact correlated, with a statistical preference for the outgoing direction having a positive projection on the incoming one [4] and recent work point at the possibility that the correlation might be regulated and exploited to increase swimming efficiency [5]. Correlations decay at any rate on the timescale of a few runs/tumbles.
} 
back) and if the difference is positive, the probability of tumbling is reduced, so that runs last longer [6]. This results in a biased random walk and a net up-gradient mean velocity.

How are time comparisons encoded at the molecular level and how does the process manage to overcome the very many sources of fluctuations present at the microscale of the bacterium?

Control of the tumbling rate is achieved via the regulation of the activity of the protein CheY: when the protein in its phosphorylated form (CheYp) hits the flagellar motor, it increases the rate of switching from counterclockwise to clockwise rotation, i.e. from run to tumble. The equilibrium between the two forms, CheY and CheYp, is governed by two enzymes: the phosphatase CheZ, which converts CheYp into CheY, and the kinase CheA, which does the opposite. Finally, the coupling with the external environment is brought by the control of the CheA activity, which is reduced when receptors are bound by chemoattractants. The net effect is that the more the receptor is bound, the less active is CheA and the higher is the probability to observe runs, via the reduced concentration of CheYp.

Another crucial feature of the pathway is constituted by the feedbacks exerted upon the sensitivity of the receptors. Receptors are well described by equilibrium two-state (active vs inactive) models [7]. The equilibrium between the two forms is controlled by their free-energy difference, which is regulated by the number of methyl groups attached to the receptor [8]. The number of methyl groups is controlled by two molecules CheR and CheB (attaching and detaching them, respectively). The two molecules provide for the integral feedback constituting the backbone of the mechanism ensuring the adaptation of the chemotaxis pathway $[9,10]$. The basic backbone is supplemented by the interaction and the cross-talk among the receptors via the so-called "assistance neighborhood" [11-13]. Interaction among the receptors is well described by the allosteric model of Monod-Wyman-Changeux, [14] which is the limiting case of the models discussed in $[15,16]$. A related subject of interest is the characterization of the variability observed across various individuals and within a single bacterium, which is an ongoing very active subject of research $[17,18]$.

Recommended papers build upon the experimental and modeling results just described and importantly advance the state-of-the-art.

The first paper recently provided a complete characterization of the adaptation module and its coupling to the rest of the network by using Fluorescence Resonance Energy Transfer (FRET) to measure intracellular responses to time-varying stimuli. Receptor sensitivity was characterized by step stimuli, the gradient sensitivity by exponential ramp stimuli and the frequency response by exponential sine-wave stimuli. A notable result is the experimental determination of the free-energy difference between the two receptor states as a function of the receptor methylation state. The difference is found to be linear, which can be shown to have fundamental implications upon the way the pathway responds to weak perturbations of the chemoattractant stimulus. I also find the paper remarkable in demonstrating how fruitful simple ideas, like the input-output relations typical of physiology (or electrical engineering), can be if properly combined with modern tools of molecular biology, such as the FRET technique. 
The second recommended paper is a very recent work that focuses on a crucial example of fluctuations, temperature, showing how they can get buffered. It shows in particular how the various individual components of the pathway get affected by temperature changes, yet they mutually compensate so that the overall performance of the network remain compensated and stable over the range of physiological temperatures. The paper combines experiments and numerical simulations of the most recent theoretical models for the pathway and establishes a remarkable example of compensatory mechanisms ensuring thermal robustness at the global level. It is a safe bet that other signal transduction pathways will be discovered in the forthcoming years to involve similar compensatory mechanisms.

\section{References}

[1] HC Berg, E. coli in Motion, Springer Verlag - New York, 2003;

[2] HC Berg, Random Walks in Biology. Princeton Univ. Press, 1993.

[3] S. Childress, Mechanics of Swimming and Flying. Cambridge Univ. Press, 1981.

[4] Turner L, Ryu WS, Berg HC. Real-time imaging of fluorescent flagellar filaments.

J Bacteriol, 182, 2793-2801, 2000.

[4] HC Berg, DA Brown. Chemotaxis in Escherichia coli analysed by three dimensional tracking. Nature, 239, 500-504, 1972.

[5] N Vladimirov, D Lebiedz \& V Sourjik Predicted Auxiliary Navigation Mechanism of Peritrichously Flagellated Chemotactic Bacteria PloS Comput Biol. 6(3): e1000717, 2010.

[6] JE Segall, SM Block \& HC Berg. Temporal comparisons in bacterial chemotaxis.

Proc Natl Acad Sci USA, 83:8987-91, 1986.

[7] S Asakura \& H Honda. Two-state model for bacterial chemoreceptor proteins. J. Mol. Biol. 176, 349-367, 1984.

[8] M Eisenbach. Chemotaxis. Imperial College Press, London, 2004.

[9] N Barkai, S Leibler. Robustness in simple biochemical networks. Nature,

387, 913-917, 1997; U Alon, N Barkai, MG Surette \& S Leibler. Adaptation in bacterial chemotaxis, Nature, 397, 168-71, 1999.

[10] TM Yi, Y Huang, MI Simon, J Doyle. Robust perfect adaptation in bacterial chemotaxis through integral feedback control. PNAS, 97, 4649-53, 2000.

[11] BA Mello \& YH Tu An allosteric model for heterogeneous receptor complexes:

Understanding bacterial chemotaxis responses to multiple stimuli. PNAS 48:17354-59, 2005.

[12] RG Endres \& NS Wingreen, Precise adaptation in bacterial chemotaxis through

"assistance neighborhoods". PNAS, 103, 13040, 2006.

[13] JE Keymer, RG Endres, M Skoge, Y Meir, NS Wingreen. Chemosensing in

Escherichia coli: Two regimes of two-state receptors. PNAS, 103, 1786-1791, 2006.

[14] G Lan, S Schulmeister, V Sourjik \& Y Tu. Adapt locally and act globally: strategy to maintain high chemoreceptor sensitivity in complex environments. Mol Syst Biol. 15, 475, 2011.

[16] J Monod, J Wyman \& JP Changeux On the nature of allosteric transitions : a plausible model. J Mol Biol. 12, 88-118, 1965.

[16] D Bray, MD Levin \& CJ Morton-Firth, Receptor clustering as a cellular mechanism to control sensitivity. Nature 393, 85-88, 1998.

[17] TAJ Duke, D Bray. Heightened sensitivity of a lattice of membrane receptors.

Proc Natl Acad Sci USA, 96:10104-108, 1999.

[17] T Emonet \& P Cluzel Relationship between cellular response and behavioral variability in bacterial chemotaxis. Proc Natl Acad Sci USA, 105, 3304-09, 2008.

[18] H Park, W Pontius, CC Guet, JF Marko, T Emonet \& P Cluzel. Interdependence of behavioural variability and response to small stimuli in bacteria. Nature, 9, 819-23. 2010. 